\title{
Financial Analysis of Dalbavancin for Acute Bacterial Skin and Skin Structure Infections for Self-Pay Patients
}

Adam G. Pizzuti (D) E. Yancy Murray · Jamie L. Wagner •

Derek A. Gaul · Christopher M. Bland · Bruce M. Jones

Received: June 25, 2020 / Accepted: September 23, 2020 / Published online: October 21, 2020

(C) The Author(s) 2020

\section{ABSTRACT}

Introduction: Acute bacterial skin and skin structure infections (ABSSSI) are an increasing cause of admission in the self-pay population. We previously reported that patients with ABSSSI discharged to receive dalbavancin showed a decreased length of stay (LOS) and total direct costs without increasing 30-day readmission rate. For patients who are financially eligible, a dalbavancin vial replacement program can offset costs. The objective of this study was to determine cost differences in treating ABSSSI in self-pay inpatients discharged to receive dalbavancin compared to standard of care (SOC).

Methods: This retrospective cohort within a community health system compared self-pay

Digital Features To view digital features for this article go to https://doi.org/10.6084/m9.figshare.12771209.

A. G. Pizzuti $(\bowtie) \cdot$ D. A. Gaul · C. M. Bland .

B. M. Jones

St. Joseph's/Candler Health System, Savannah, GA, USA

e-mail: adam.pizzuti@prismahealth.org

A. G. Pizzuti · E. Y. Murray · D. A. Gaul ·

C. M. Bland · B. M. Jones

University of Georgia College of Pharmacy,

Savannah, GA, USA

J. L. Wagner

University of Mississippi School of Pharmacy,

Jackson, MS, USA adult inpatients with ABSSSI from February 3, 2016 to August 5, 2019 discharged to receive dalbavancin at an outpatient infusion center with SOC intravenous antibiotics. Patients were included with cellulitis, abscess, or postoperative wound infections diagnoses on the basis of International Classification of Disease, Tenth Revision (ICD-10) codes. Excluded populations were patients without dalbavancin vial replacement performed, pregnant, infections caused exclusively by gram-negative bacteria or fungi, or ICD-10 codes not consistent with ABSSSI. The primary outcome was direct cost of hospital stay. Secondary outcomes included length of stay (LOS), 30-day readmission rates, adverse events (AE), and indirect hospital costs. On the basis of previous studies, a one-sided Student's $t$ test was performed on financial data. Results: Twelve dalbavancin and 263 SOC patients met inclusion criteria. Direct cost ( $\$ 2758$ vs $\$ 4010, p=0.105)$ and indirect hospital cost ( $\$ 2913$ vs $\$ 3646, p=0.162)$ per patient were less in the dalbavancin group. There was no significant difference between median LOS ( 4 vs $4, p=0.888)$, $\mathrm{AE}(0 \%$ vs $14.8 \%$ ), and 30 -day readmission rates for dalbavancin vs SOC group $(8.3 \%$ vs $7.2 \%$, $p=0.604$ ).

Conclusion: Self-pay patients with ABSSSI discharged to receive dalbavancin with vial replacement resulted in decreased direct and indirect costs per patient with similar 30-day readmission rates, $\mathrm{AE}$, and $\mathrm{LOS}$. More studies 
targeted toward this population are warranted to determine ultimate benefit.

Keywords: ABSSSI; Dalbavancin; Financial analysis; Self-pay; Vial replacement

\section{Key Summary Points}

Acute bacterial skin and skin structure infections (ABSSSI) are an increasing cause of admission in the self-pay population.

Dalbavancin, a novel lipoglycopeptide, has been shown to decrease length of stay (LOS) and total direct costs without increasing 30-day readmission rate.

The objective of this study was to examine the potential cost savings in treating selfpay inpatients with ABSSSI discharged to receive dalbavancin compared to standard of care (SOC).

Self-pay patients with ABSSSI discharged to receive dalbavancin with vial replacement resulted in decreased direct and indirect costs per patient with similar 30-day readmission rates, $\mathrm{AE}$, and LOS.

More studies targeted toward this population are warranted to determine ultimate benefit.

\section{DIGITAL FEATURES}

This article is published with digital features, including a summary slide, to facilitate understanding of the article. To view digital features for this article go to https://doi.org/10.6084/ m9.figshare.12771209.

\section{INTRODUCTION}

Acute bacterial skin and skin structure infections (ABSSSI) are defined by the US Food and Drug Administration (FDA) as a bacterial infection of the skin with erythema, tenderness, warmth, or swelling. The lesion size should be at least $75 \mathrm{~cm}^{2}$, measured by the area of redness, edema, or induration. These include cellulitis/ erysipelas, wound infections, and major cutaneous abscesses. The most common causative pathogens are Streptococcus pyogenes and Staphylococcus aureus, including methicillin-resistant $S$. aureus (MRSA) $[1,2]$. ABSSSI are common reasons for presenting to doctor's offices and hospitals. While many low-risk patients who present to the emergency department are able to be treated as outpatients, hospital admissions for ABSSSI continue to rise [3-5].

According to the US Census Bureau, in 2018, 27.5 million (8.5\%) people were classified as uninsured (i.e., self-pay), which was an increase of $0.5 \%$ from 2017 [6]. Of these, the majority $(13.8 \%)$ are classified as having the lowest income (less than $\$ 25,000$ US dollars household income) $[6,7]$. While this is but a fraction of the total US population, hospitals risk having to cover uncompensated healthcare costs on their own with this patient population. According to the American Hospital Association, in 2017, community hospitals provided $\$ 38.4$ billion in uncompensated care cost [8]. Understanding the financial impact of treating this patient population could improve financial decisionmaking.

ABSSSI has been increasing as a cause for admissions in the self-pay patient population [9]. In a previous study conducted at our institution, dalbavancin resulted in decreased LOS and 30-day readmission rate compared to national data for patients with ABSSSI. While this ultimately led to a reduction in cost for the healthcare system, it included different patient socioeconomic statuses [10]. Dalbavancin, a long-acting lipoglycopeptide, is FDA indicated to treat adult patients with ABSSSI caused by susceptible isolates of the following gram-positive microorganisms: S. aureus (including MRSA), S. pyogenes, Streptococcus agalactiae, and the Streptococcus anginosus group. It is a 30-min, single intravenous (IV) infusion that is an alternative for the treatment of ABSSSI [11, 12]. Previous studies with dalbavancin have shown reductions in length of stay (LOS) and inpatient cost for ABSSSI [13-18]. Additionally, the manufacturer of dalbavancin has a patient assistance 
program that provides the drug at no cost through vial replacement for patients who are financially eligible (including patients who are uninsured or underinsured and unable to afford the cost of therapy) $[19,20]$. After reviewing the current available literature, there are no current data on the self-pay population who receive dalbavancin outpatient compared to traditional inpatient treatment of ABSSSI. The aim of this study was to determine if there is a cost difference in treatment of ABSSSI in self-pay patients discharged and treated as an outpatient with dalbavancin compared to traditional inpatient IV antibiotic treatment, standard of care (SOC).

\section{METHODS}

This study was conducted under institutional review board approval from St. Joseph's/Candler Health System which includes two hospitals and one outpatient infusion center included in the study. In addition, this study adheres to the declaration of Helsinki of 1964 and its later amendments.

The current process for receipt of dalbavancin began in February of 2016. A retrospective cohort was compiled via a computergenerated report from February 3, 2016 to August 5, 2019. Patients admitted with an ABSSSI were collected into two groups: one that received dalbavancin after discharge and a SOC group with no intervention for dalbavancin. In the dalbavancin group, patients were selected on the basis of evaluation from the infectious diseases pharmacist (ID PharmD) or the physician. This evaluation targets specific patient populations that may benefit from dalbavancin therapy (e.g., limited comorbidities, failed outpatient antibiotics, IV drug users (IVDU)). All patients that were admitted for IV antibiotic therapy were analyzed using systemic inflammatory response syndrome criteria (temperature $>38^{\circ} \mathrm{C}$ or $<36{ }^{\circ} \mathrm{C}$, heart rate $>90$ beats/ min, respiratory rate $>20$ breaths $/ \mathrm{min}$, and white blood cell count $>12,000$ cells/dL or $<4000$ cells/dL) as a reference to aid the clinician to determine if the patient was a candidate for dalbavancin therapy and determine if the patient was medically ready for discharge.
Factors also evaluated for every patient were if the patient was immunocompetent, hemodynamically stable, afebrile, had a normal (baseline) mental status, and willingness to consent to this therapy option. The process involved a multidisciplinary collaborative approach including the physician, ID PharmD, case manager, and infusion center patient assistance coordinator [10]. Upon discharge, self-pay, consenting patients received dalbavancin at the health system's on-site, outpatient infusion center and the healthcare team applied for vial replacement to assist with cost of therapy.

Patients were included if they were adult inpatients admitted with self-pay insurance status (i.e., uninsured) and an ABSSSI (International Classification of Disease, Tenth Revision (ICD-10) codes L03.XXX, L02.XXX, T81.XXX consistent with cellulitis, abscess, or postoperative wound infection, respectively). Patients were excluded if they were pregnant, did not have dalbavancin vial replacement performed, had infections caused exclusively by gram-negative bacteria or fungi, or ICD-10 codes consistent with M86.XXX, M72.6, A48.0, E08.621/ E09.521/E10.621/E11.621/E13.621, representing osteomyelitis, necrotizing fasciitis, gangrene, or diabetic foot ulcer, respectively. Background demographics including infectious diagnosis, comorbid disease states, allergies, hemodynamic status, renal and liver function, and microbiology data were collected for study subjects.

The primary outcome was total direct cost of hospital stay, which was recorded as the total direct cost per patient. The secondary outcomes evaluated LOS, 30-day readmission rates, adverse events (AE) during hospitalization, and indirect costs of hospital stay. Direct costs consisted of any monetary value attributed directly to the treatment of infection with IV antibiotics during the patients' inpatient stay. This included costs associated with medical supplies, laboratory, pharmacy, emergency department, operating room, room stay, and salaries related to medical personnel (e.g., nurses) directly providing patient care. Indirect costs consisted of monetary values that were not directly related to that specific department (e.g., overhead building costs or hospital administration cost). 
Costs are allocated monthly at the host facility to each specific department after reports collect the financial charges. Data was collected and analyzed in Microsoft ${ }^{\circledR}$ Office Excel. Categorical data were assessed using chi-square or Fisher's exact test, as appropriate. Continuous data were assessed using Student's $t$ test or Mann-Whitney $U$, as appropriate. An alpha of 0.05 was deemed statistically significant. A one-sided Student's $t$ test was performed on the financial data owing to previous aforementioned studies suggesting the cost-savings effects of dalbavancin making an alpha of 0.025 statistically significant.

\section{RESULTS}

A total of 397 self-pay inpatients with ABSSSI were identified from February 3, 2016 to August 5, 2019. After applying exclusion criteria, 12 patients were discharged and received dalbavancin with patient assistance and receipt of vial replacement and were included in the dalbavancin group and 263 patients received normal course of care and were included in the SOC group (Fig. 1). Patient demographics and clinical characteristics are summarized in Table 1 . The mean age was around 42 years old in both groups $(p=0.986)$ and patients were typically male $(171 / 275,62.3 \%)$ with a higher percentage of male patients in the dalbavancin group. Additional common patient demographics included in this study were Caucasian race $(174 / 275,63.3 \%)$ and diagnosis of cellulitis $(186 / 275,67.6 \%)$. Hyperlipidemia $(25.0 \%$ vs $5.0 \%, p=0.026)$, cancer $(16.7 \%$ vs $1.9 \%$, $p=0.033$ ), and peripheral artery disease (PAD) $(8.3 \%$ vs $0, p=0.044)$ were statistically higher in the dalbavancin group compared to SOC. The most common causative organism isolated in the dalbavancin group was $S$. aureus $(7 / 7,100 \%)$ compared to $58.6 \%$ in the SOC group $(p=0.047)$. The majority of the positive cultures were wound cultures in both the dalbavancin and SOC groups, $58.3 \%$ and $51.3 \%$, respectively. All patients (100\%) in the dalbavancin group received vancomycin empirically compared to $81.7 \%$ in the SOC group $(p=0.135)$. Piperacillin/tazobactam, cefazolin, and clindamycin IV were the next most common empiric therapy given.

The primary and secondary outcomes are displayed in Table 2 . The primary outcome demonstrated that dalbavancin was associated with a lower total direct cost per patient compared to SOC ( $\$ 2758$ vs $\$ 4010$, respectively, $p=0.105)$. This resulted in dalbavancin shown as a cost-saving option of $\$ 1252$ per patient. Focusing on secondary outcomes, the median length of stay was similar in both groups (4 days; $p=0.888$ ) with similar readmission rates in the dalbavancin vs SOC groups $(1 / 12$, $8.3 \%$ vs $19 / 263,7.2 \% ; p=0.604$, respectively). There were no statistically significant differences for 30-day readmission rates, acute kidney injury, or hospital acquired infections. Indirect costs per patient were numerically lower in the dalbavancin group ( $\$ 2913$ vs $\$ 3646 ; p=0.162$ ).

\section{DISCUSSION}

Overall this study demonstrated discharging self-pay patients with a diagnosis of ABSSSI to receive dalbavancin and patient assistance through vial replacement to be an effective costsaving strategy. From 2005 to 2014 skin and subcutaneous tissues infections in adults aged 18-44 years have been documented in the top five reasons for hospital admission [21]. With interventions in hospitals being focused on patient outcomes that are clinically safe, effective, and cost conscientious, understanding the financial impact of treatment options is important in this common inpatient diagnosis [22]. This study found dalbavancin to have no difference in clinical outcomes or safety profile based on no significant increase in LOS, 30-day readmission rates, or adverse events while in the hospital. In addition to dalbavancin being shown as a clinically effective agent for ABSSSI in self-pay patients, this study also highlights the cost-savings aspect of choosing this therapy which is discussed in more detail in paragraphs below.

This study found that patients with ABSSSI were consistent with patient characteristics in previous studies analyzing the use of dalbavancin regarding age, gender, race, and 


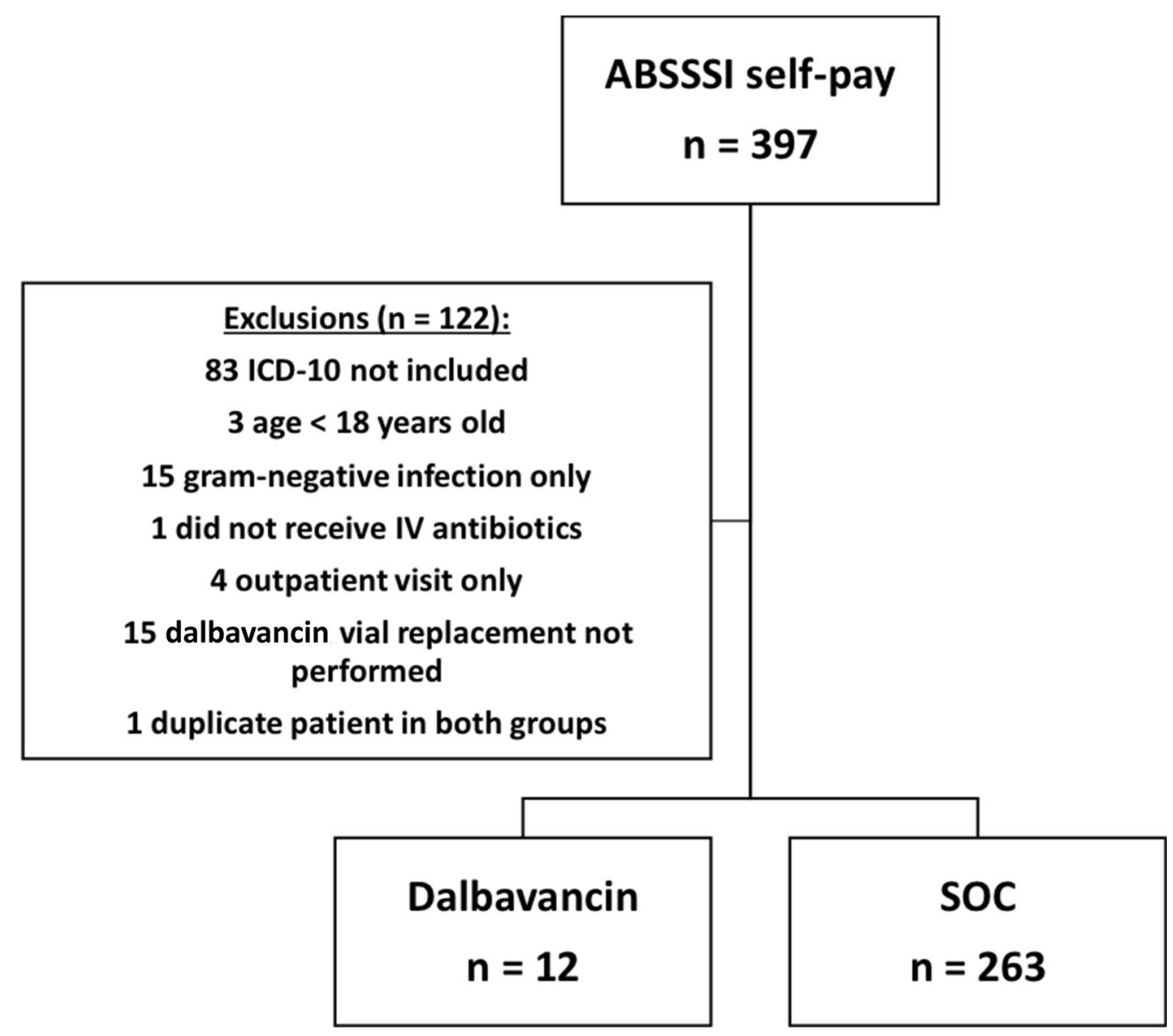

Fig. 1 Patient enrollment breakdown

infectious diagnosis [13-18]. Streifel et al. [13] found that patients treated with dalbavancin in their academic center had a mean age of 48.6 years with $22 \%$ of the infections being skin and soft-tissue infections, only second behind osteomyelitis. Morrisette et al. [14] found similar patients in their analysis of long-acting lipoglycopeptides for ABSSSI. The majority of the ABSSSI cases were cellulitis. This was consistent with previous data showing cellulitis and/or abscess making up $80 \%$ of the cases while $20 \%$ had wound infections (e.g., posttraumatic or postoperatively) [23]. There was no difference found in antibiotic allergy status. This is important to note as certain allergies (e.g., penicillin) have been linked to increased rates on antibiotic resistance, Clostridioides difficile, and increased use of broad-spectrum antibiotics which could have influenced the primary and secondary outcomes [24, 25]. A numerically higher amount of IVDU in the dalbavancin group were found (25\% vs $13.7 \%$, $p=0.387$ ). This was similar to the finding in the study by Morrisette et al. [14] that also discovered the high percentage of patients who are IVDUs with methicillin-susceptible and methicillin-resistant $S$. aureus infections are eligible for dalbavancin. Outpatient parenteral antimicrobial therapy (OPAT), specifically long-acting lipoglycopeptides, pose as a useful solution in this patient population. There are many advantages to this treatment option, especially with dalbavancin. These include a reduced need for long-term central lines which reduces infection risk and substance abuse concerns, increased compliance for patients with poor adherence or difficult life situations (e.g., homelessness, incarceration, rural location) 
Table 1 Patient clinical and microbiological characteristics

\begin{tabular}{|c|c|c|c|}
\hline Variables & Dalbavancin $(n=12)$ & SOC $(n=263)$ & $p$ value \\
\hline Age, years $($ mean $\pm S D)$ & $41.75 \pm 6.3$ & $41.71 \pm 11.6$ & 0.986 \\
\hline Sex, male & $12(100)$ & $159(60.5)$ & 0.006 \\
\hline \multicolumn{4}{|l|}{ Race } \\
\hline Caucasian & $11(91.7)$ & $163(62)$ & 0.061 \\
\hline African American & $1(8.3)$ & $92(35)$ & $<0.001$ \\
\hline Pacific Islander or unknown origin & $0(0)$ & $8(3)$ & 1.000 \\
\hline \multicolumn{4}{|l|}{ ICD-10 } \\
\hline Cellulitis (L03.XXX) & $9(75)$ & $177(67.3)$ & 0.757 \\
\hline Abscess (L02.XXX) & $3(25)$ & $79(30)$ & 0.002 \\
\hline Postop wound infections (T81.XXX) & $0(0)$ & $7(2.7)$ & 1.000 \\
\hline \multicolumn{4}{|l|}{ Antibiotic allergies } \\
\hline Total & $1(8.3)$ & $65(24.7)$ & 0.305 \\
\hline Penicillin & $0(0)$ & $30(11.4)$ & 0.374 \\
\hline Sulfonamide & $1(8.3)$ & $17(6.5)$ & 0.564 \\
\hline Clindamycin & $0(0)$ & $5(1.9)$ & 1.000 \\
\hline Vancomycin & $0(0)$ & $5(1.9)$ & 1.000 \\
\hline Other & $0(0)$ & $8(3.0)$ & 1.000 \\
\hline \multicolumn{4}{|l|}{ Vitals } \\
\hline BMI, $\mathrm{kg} / \mathrm{m}^{2}$ & $29.15[26.9-38.7]$ & $28.89[23.5-35.4]$ & 0.555 \\
\hline Initial $\mathrm{BMI} \geq 30 \mathrm{~kg} / \mathrm{m}^{2}$ & $4(33.3)$ & $109(41.4)$ & 0.766 \\
\hline Maximum temperature in $24 \mathrm{~h},{ }^{\circ} \mathrm{F}$ & $98.85[98.5-99.8]$ & $98.9[98.5-100.1]$ & 0.761 \\
\hline Maximum temperature in $24 \mathrm{~h},>100.4^{\circ} \mathrm{F}$ & $2(16.7)$ & $65(24.7)$ & 0.736 \\
\hline$\geq 2(\mathrm{RR} \geq 22, \mathrm{SBP} \leq 100)$ & $0(0)$ & $1(0.38)$ & 1.000 \\
\hline Maximum WBC in $24 \mathrm{~h}$, cells $/ \mathrm{mm}^{3}$ & $11.15[9.4-15.3]$ & $10.90[8.7-15.1]$ & 0.722 \\
\hline Maximum WBC in $24 \mathrm{~h} \geq 12 \mathrm{cells} / \mathrm{mm}^{3}$ & $4(33.3)$ & $114(43.4)$ & 0.564 \\
\hline \multicolumn{4}{|l|}{ Comorbidities } \\
\hline Diabetes & $2(16.7)$ & $53(20.2)$ & 1.000 \\
\hline Hypertension & $3(25)$ & $73(27.8)$ & 1.000 \\
\hline Hyperlipidemia & $3(25)$ & $13(4.9)$ & 0.026 \\
\hline Cancer & $2(16.7)$ & $5(1.9)$ & 0.033 \\
\hline Venous thromboembolism & $2(16.7)$ & $10(3.8)$ & 0.090 \\
\hline Peripheral artery disease & $1(8.3)$ & $0(0)$ & 0.044 \\
\hline
\end{tabular}


Table 1 continued

\begin{tabular}{|c|c|c|c|}
\hline Variables & Dalbavancin $(n=12)$ & SOC $(n=263)$ & $p$ value \\
\hline Chronic kidney disease & $0(0)$ & $4(1.5)$ & 1.000 \\
\hline Hepatitis & $1(8.3)$ & $13(4.9)$ & 0.473 \\
\hline Cirrhosis & $0(0)$ & $3(1.1)$ & 1.000 \\
\hline ABSSSI history documented & $4(33.3)$ & $59(22.4)$ & 0.480 \\
\hline History of Clostridioides difficile & $1(8.3)$ & $3(1.1)$ & 0.164 \\
\hline IVDU & $3(25)$ & $36(13.7)$ & 0.387 \\
\hline Recreation drug abuse (non-IV) & $3(25)$ & $59(22.4)$ & 0.736 \\
\hline Smoker & $8(66.7)$ & $144(54.8)$ & 0.557 \\
\hline ID consult & $10(37)$ & $126(47.9)$ & 0.019 \\
\hline \multicolumn{4}{|l|}{ Microbiological data } \\
\hline Cultures obtained & $12(100)$ & $234(89)$ & 0.622 \\
\hline Wound culture & $8(30.8)$ & $148(29.7)$ & 0.562 \\
\hline Abscess culture & $4(15.4)$ & $105(21.1)$ & 0.769 \\
\hline Blood culture & $10(38.5)$ & $189(38)$ & 0.521 \\
\hline Urine culture & $4(15.38)$ & $56(11.24)$ & 0.301 \\
\hline Positive culture & $7(58.3)$ & $128(54.7)$ & 0.566 \\
\hline S. aureus infection & $7(100)$ & $75(58.6)$ & 0.047 \\
\hline MRSA & $4(57.1)$ & $39(52)$ & 0.100 \\
\hline MSSA & $3(42.9)$ & $35(46.7)$ & 0.221 \\
\hline Blood culture & $0(0)$ & $21(9)$ & 0.608 \\
\hline Wound culture & $7(58.3)$ & $120(51.3)$ & 0.556 \\
\hline Urine culture & $0(0)$ & $11(4.7)$ & 1.000 \\
\hline \multicolumn{4}{|l|}{ Inpatient antibiotics } \\
\hline Vancomycin & $12(100)$ & $215(81.7)$ & 0.135 \\
\hline Piperacillin/tazobactam & $6(50)$ & $162(61.6)$ & 0.547 \\
\hline Meropenem & $0(0)$ & $1(0.4)$ & 1.000 \\
\hline Cefazolin & $4(33.3)$ & $41(15.6)$ & 0.114 \\
\hline Clindamycin (IV/PO) & $3(25)$ & $143(54.4)$ & 0.073 \\
\hline Other & $3(25)$ & $102(38.8)$ & 0.545 \\
\hline
\end{tabular}

Data are presented as $n(\%)$ or median [IQR] unless otherwise indicated

$B M I$ body mass index, $R R$ respiratory rate, $S B P$ systolic blood pressure, $I V D U$ intravenous drug users, $I D$ infectious diseases, MRSA methicillin-resistant $S$. aureus, MSSA methicillin-susceptible $S$. aureus, $P O$ oral 
Table 2 Clinical outcomes

\begin{tabular}{|c|c|c|c|}
\hline Variables & $\begin{array}{l}\text { Dalbavancin } \\
(n=12)\end{array}$ & $\begin{array}{l}\text { SOC } \\
(n=263)\end{array}$ & $p$ value \\
\hline \multicolumn{4}{|l|}{ Primary outcome } \\
\hline $\begin{array}{l}\text { Total direct costs, } \\
\text { US\$ per patient }\end{array}$ & $\$ 2758$ & $\$ 4010$ & 0.105 \\
\hline \multicolumn{4}{|l|}{ Secondary outcomes } \\
\hline $\begin{array}{l}\text { Total indirect } \\
\text { cost, US\$ per } \\
\text { patient }\end{array}$ & $\$ 2913$ & $\$ 3646$ & 0.162 \\
\hline $\begin{array}{l}\text { Baseline serum } \\
\text { creatinine, } \mathrm{mg} / \mathrm{dL}\end{array}$ & $0.8[0.7-1]$ & $\begin{array}{l}0.9 \\
\quad[0.7-1]\end{array}$ & 0.640 \\
\hline $\begin{array}{l}\text { Acute kidney } \\
\text { injury }\end{array}$ & $0(0)$ & $33(12.6)$ & 0.371 \\
\hline $\begin{array}{l}\text { Hospital acquired } \\
\text { infection }\end{array}$ & $0(0)$ & $2(0.8)$ & 1.000 \\
\hline $\begin{array}{l}\text { Documented } \\
\text { infusion reaction }\end{array}$ & $0(0)$ & $4(1.5)$ & 1.000 \\
\hline $\begin{array}{l}\text { Median length of } \\
\text { stay, days }\end{array}$ & $4[2.8-4.3]$ & $4[2-6]$ & 0.888 \\
\hline $\begin{array}{l}\text { 30-day } \\
\text { readmission rate }\end{array}$ & $1(8.3 \%)$ & $19(7.2 \%)$ & 0.604 \\
\hline
\end{tabular}

Data are presented as $n(\%)$ or median [IQR]

with a one-time dose, and discharge assistance when oral antibiotics may not be adequate treatment [15].

Interestingly, there were a high amount of infectious diseases (ID) consults in this population, with a higher percentage in the SOC group ( $37 \%$ vs $48 \%$ ). This could be attributed to the underlying difficult transitions of care decisions with a self-pay patient population [26]. All of the patients discharged to receive dalbavancin in this study were empirically started on vancomycin at admission. This could be explained as vancomycin is one of the targeted antimicrobial therapies reviewed by the ID PharmD on a daily basis and the difficulties in identifying possible dalbavancin candidates in a reasonable timeframe. The increased risk of side effects and lack of alternate treatment options lend to the ID PharmD being able to determine potential dalbavancin candidates and assist with transitions of care [27]. The impact of the ID PharmD has been shown in the previous study, as they are able to assist with clinical knowledge skills to aid in selecting, counseling, and effectively treating this patient population with dalbavancin [10].

Results showed discharging to receive dalbavancin for ABSSSI to be a cost-saving option of $\$ 1252$ per patient. Previous studies have shown both cost and LOS reduction benefits with dalbavancin. Streifel et al. [13] found that analyzing 27 patients who received dalbavancin found an avoidance of 617 hospital days which resulted in a mean cost savings per patient of $\$ 40,414$; however, that study did include nonABSSSI cases. An additional study in Germany demonstrated the cost-savings effect of dalbavancin in MRSA ABSSSI by reducing LOS by 6.45 days with a cost savings of $\$ 3099.69$ $(2865 €)$ [16]. The previous study at our institution was associated with decreased cost and length of stay [10]. The impact of vial replacement was shown to mirror previous data of dalbavancin as a cost-effective option in the self-pay population. To our knowledge, there are no current data to compare the financial results to self-pay patients. For those with thirdparty payers, dalbavancin has been shown to save $\$ 1442$ to $\$ 4803$ per complicated skin and soft-tissue infection (cSSTI) case with a safe adverse event profile [28].

In an economic evaluation of dalbavancin in three European countries, dalbavancin was found to reduce length of stay for patients with ABSSSI by 3.3 days per patient with no additional cost towards the health system [17]. Additionally, McCarthy et al. [18] found a reduction in length of stay (3.2 days vs 4.8 days, $p=0.003$ ) which was attributed to an increase in productivity for the staff. While this study did not result in a significant decrease in length of stay, there were two confounding factors that could have contributed to this. One patient in the dalbavancin group had a LOS of 11 days. There was an attempt to adjust for this by presenting the data as median days. Additionally, for ABSSSI there was a high percentage in the 
standard of care group of ID consults (47.9\%), which could have contributed to a shorter length of stay. The adverse event profile was similar in this study between the two groups. This was important since previous literature has shown MRSA ABSSSI treated with vancomycin has an increased risk for acute kidney injury (AKI) in patients with no medical insurance (OR 3.451, 95\% CI 1.131-9.090) [27]. The rates of AKI in both treatment groups were similar despite $50 \%$ of MRSA infections in both groups.

Several limitations are present within this study. First, the low number of patients enrolled in the dalbavancin arm $(n=12)$ makes it difficult to determine definitive conclusions on safety and efficacy. Similarly, the retrospective nature of this design limited the ability to control factors, such as patient assistance and vial replacement receipt. The 15 patients that did not have vial replacement performed identify a performance improvement for our institution but a limitation of this study. Additionally, these patients were not randomized. This led to certain comorbidity and infection etiology discrepancies that could have influenced clinical parameters outside of costs. During the screening process for patients, it was found that some patients had a documented abscess infection without a matching ICD-10 code. This potential human error could have resulted in fewer patients being included in the two groups and lower numbers in the abscess groups. Some patients who met inclusion criteria might not have been candidates for dalbavancin therapy. This is because only primary diagnosis codes for inclusion or exclusion criteria were used. Secondary codes could have influenced LOS or prohibited use of dalbavancin therapy. Access to the financial data was limited because some accounts were frozen for financial reimbursement investigation or deemed charity. Indirect costs were limited to data on current inpatient stay and do not reflect any outpatient costs (i.e., outpatient antibiotic prescriptions) associated with care beyond dalbavancin patients receiving infusion.

\section{CONCLUSION}

These findings suggest that discharging self-pay patients diagnosed with an ABSSSI to receive dalbavancin at an infusion center is less expensive than the inpatient standard of care for treating self-pay patients with ABSSSI. Although this study did not show a decrease in LOS or 30-day readmission rate similar to previous studies, it did not show an increase. Future analysis could include a prospective analysis consisting of a larger number of selfpay dalbavancin patients ensuring vial replacement is performed. These findings help provide a guide for community hospitals for selecting and making financially sound decisions for treating patients with ABSSSI with dalbavancin.

\section{ACKNOWLEDGEMENTS}

Funding. No funding or sponsorship was received for this study or publication of this article.

Authorship. All named authors meet the International Committee of Medical Journal Editors (ICMJE) criteria for authorship for this article, take responsibility for the integrity of the work as a whole, and have given their approval for this version to be published.

Prior Presentation. Preliminary data was presented on April 28, 2020 at the virtual Georgia Society of Health-System Pharmacists Spring meeting.

Disclosures. Christopher M. Bland is on the Speaker's Bureau for Merck and Tetraphase, on the Advisory Board for Merck and Paratek, and has received grant funding from ALK-Abello. Bruce M. Jones is on the Speaker's Bureau for Allergan, Tetraphase, and Paratek and has received grant funding from and participates in consulting for ALK-Abello. E. Yancey Murray, Derek A. Gaul, and Jamie L. Wagner have no conflicts of interest. Adam G. Pizzuti is now affiliated with Prisma Health Midlands, Columbia, SC, USA. 
Compliance with Ethics Guidelines. This study received IRB-approval from St. Joseph's/ Candler Health system. In addition, this study adheres to the declaration of Helsinki of 1964 and its later amendments.

Data Availability. All datasets are made available in this published article and are available from the corresponding author on reasonable request.

Open Access. This article is licensed under a Creative Commons Attribution-NonCommercial 4.0 International License, which permits any non-commercial use, sharing, adaptation, distribution and reproduction in any medium or format, as long as you give appropriate credit to the original author(s) and the source, provide a link to the Creative Commons licence, and indicate if changes were made. The images or other third party material in this article are included in the article's Creative Commons licence, unless indicated otherwise in a credit line to the material. If material is not included in the article's Creative Commons licence and your intended use is not permitted by statutory regulation or exceeds the permitted use, you will need to obtain permission directly from the copyright holder. To view a copy of this licence, visit http://creativecommons.org/licenses/by$\mathrm{nc} / 4.0 /$.

\section{REFERENCES}

1. Food and Drug Administration. Guidance for industry acute bacterial skin and skin structure infections: developing drugs for treatment. FDA. 2013. https://www.fda.gov/media/71052/ download. Accessed 30 Jan 2020.

2. Stevens DL, Bisno AL, Chambers HF, et al. Practice guidelines for the diagnosis and management of skin and soft tissue infections update by the Infectious Diseases Society of America. Clin Infect Dis. 2014;59:e10-52.

3. Bookstaver PB, Jenkins TC, Stenehjem E, et al. Impact of outpatient vs inpatient ABSSSI treatment on outcomes: a retrospective observational analysis of medical charts across US Emergency Departments. Open Forum Infect Dis. 2018;5(7):ofy109.
4. Bork JT, Heil EL, Berry S, et al. Dalbavancin use in vulnerable patients receiving outpatient parenteral antibiotic therapy for invasive gram-positive infections. Infect Dis Ther. 2019;8:171-84.

5. Kaye KS, Patel DA, Stephens JM, Khachatryan A, Patel A, Johnson K. Rising United States hospital admissions for acute bacterial skin and skin structure infections: recent trends and economic impact. PLoS One. 2015;10(11):e0143276. https://doi.org/ 10.1371/journal.pone.0143276.

6. Nagamine M, Stocks C, Merrill C. Trends in uninsured hospital stays, 1998-2007: Statistical Brief \#88. 2010. In: Healthcare cost and utilization project (HCUP) statistical briefs [Internet]. Rockville (MD): Agency for Healthcare Research and Quality (US); 2006.

7. Cohen RA, Terlizzi EP, Martinez ME. Health insurance coverage: early release of estimates from the National Health Interview Survey, 2018. Hyattsville (MD): National Center for Health Statistics. 2019. https://www.cdc.gov/nchs/data/nhis/earlyrelease/ insur201905.pdf. Accessed 31 Jan 2020.

8. American Hospital Association. Uncompensated hospital care cost fact sheet. 2019. https://www. aha.org/system/files/2019-01/uncompensated-carefact-sheet-jan-2019.pdf. Accessed 12 Sep 2019.

9. Nagamine M, Stocks C, Merrill C. Trends in uninsured hospital stays, 1998-2007: Statistical Brief\# 88. 2006. https://www.ncbi.nlm.nih.gov/books/ NBK53604/. Accessed 30 Jan 2020.

10. Jones BM, Hersey R, Taylor C, Bland CM. Evaluation of dalbavancin on length of stay in acute bacterial skin and skin structure infections. J Am Coll Clin Pharm. 2019. https://doi.org/10.1002/ jac5.1085.

11. Dalbavancin [package insert]. Parsippany, NJ: Durata Therapeutics, Inc.; 2014. Available from: https://www.accessdata.fda.gov/drugsatfda_docs/ label/2014/021883s000lbl.pdf. Accessed 20 Sept 2019.

12. Dunne MW, Puttagunta S, Giordano P, Krievins D, Zelasky M, Baldassarre J. A randomized clinical trial of single-dose versus weekly dalbavancin for treatment of acute bacterial skin and skin structure infection. Clin Infect Dis. 2016;625:545-551.

13. Streifel AC, Sikkab MK, Bowena CD, Lewis JS II. Dalbavancin use in an academic medical centre and associated cost savings. Int J Antimicrobial Agents. 2019;54:652-654.

14. Morrisette T, Miller MA, Montague BT, Barber GR, McQueen RB, Krsak M. On- and off-label utilization of dalbavancin and oritavancin for Gram-positive 
infections. J Antimicrob Chemother. 2019;74(8): 2405-16.

15. Krsak M, Morrisette T, Miller M, et al. Advantages of outpatient treatment with long-acting lipoglycopeptides for serious gram-positive infections: a review. Pharmacotherapy. 2020. https://doi.org/10. 1002/phar.2389.

16. Wilke M, Worf K, Preisendörfer B, Heinlein W, Kast T, Bodmann KF. Potential savings through singledose intravenous Dalbavancin in long-term MRSA infection treatment-a health economic analysis using German DRG data. GMS Infect Dis. 2019;2019:7.

17. Marcellusi A, Viti R, Sciattella P, et al. Economic evaluation of the treatment of acute bacterial skin and skin structure infections (ABSSSIs) from the national payer perspective: introduction of a new treatment to the patient journey. A simulation of three European countries. Expert Rev Pharmacoecon Outcomes Res. 2019;19(5):581-599.

18. McCarthy MW, Keyloun KR, Gillard P, et al. Dalbavancin reduces hospital stay and improves productivity for patients with acute bacterial skin and skin structure infections: the ENHANCE trial. Infect Dis Ther. 2020;9(1):53-67.

19. Dalbavancin Website. Coding, coverage, and reimbursement. 2019. https://www.dalvance.com/ coding-and-reimbursement. Accessed 30 Jan 2020.

20. Dalbavancin. Needy meds medication assistance. 2020. https://www.needymeds.org/generic-drug/ name/dalbavancin. Accessed 25 Mar 2020.

21. McDermott, KW, Anne E, Ruirui S. Trends in hospital inpatient stays in the United States, 2005-2014. HCUP Stat Brief. 2017;2017:225. https://www.hcup-us.ahrq.gov/reports/statbriefs/ sb225-Inpatient-US-Stays-Trends.pdf.

22. Grol R, Grimshaw J. From best evidence to best practice: effective implementation of change in patients' care. Lancet. 2003;362(9391):1225-300.

23. Pulido-Cejudo A, Guzmán-Gutierrez M, JalifeMontaño A, et al. Management of acute bacterial skin and skin structure infections with a focus on patients at high risk of treatment failure. Therapeutic Adv Infect Dis. 2017;4(5):143-161.

24. Shenoy ES, Macy E, Rowe T, Blumenthal KG. Evaluation and management of penicillin allergy: a review. JAMA. 2019;321(2):188-99.

25. Jones BM, Avramovski N, Concepcion AM, Crosby $\mathrm{J}$, Bland CM. Clinical and economic outcomes of penicillin skin testing as an antimicrobial stewardship initiative in a community health system. Open Forum Infect Dis. 2019;6(4):ofz109.

26. Bhavan KP, Brown LS, Haley RW. Self-administered outpatient antimicrobial infusion by uninsured patients discharged from a safety-net hospital: a propensity-score-balanced retrospective cohort study. PLoS Med. 2015;12(12):e1001922. https:// doi.org/10.1371/journal.pmed.1001922.

27. Jorgensen SCJ, Murray KP, Lagnf AM, et al. A multicenter evaluation of vancomycin-associated acute kidney injury in hospitalized patients with acute bacterial skin and skin structure infections. Infect Dis Ther. 2020;9:89-106.

28. Agarwal R, Bartsch SM, Kelly BJ, et al. Newer glycopeptide antibiotics for treatment of complicated skin and soft tissue infections: systematic review, network meta-analysis and cost analysis. Clin Microbiol Infect. 2018;24(4):361-368. 